\title{
Therapeutic Effects of Highly Purified De-Glycosylated Gcmaf in the Immunotherapy of Patients with Chronic Diseases
}

\author{
${ }^{1}$ Lynda Thyer, ${ }^{1}$ Emma Ward, ${ }^{1}$ Rodney Smith, ${ }^{2}$ Jacopo J.V. Branca, \\ ${ }^{2}$ Gabriele Morucci, ${ }^{2}$ Massimo Gulisano, ${ }^{3}$ David Noakes and ${ }^{2}$ Stefania Pacini \\ ${ }^{1}$ Macro Innovations Ltd, Cambridge, UK \\ ${ }^{2}$ Department of Experimental and Clinical Medicine, University of Firenze, Italy \\ ${ }^{3}$ Immuno Biotech Ltd, Guernsey, Channel Islands
}

Received 2013-07-02, Revised 2013-07-15; Accepted 2013-07-24

\begin{abstract}
The de-Glycosylated vitamin D binding protein is a powerful Macrophage Activating Factor (GcMAF) that shows multiple biological effects that could be exploited in the immunotherapy of tumours, viral infections and autism. Here we report the observation of a series of clinical cases describing the results obtained administering highly purified GcMAF to patients with diverse types of chronic diseases. These are heterogeneous and refer to patients with different types of diseases at different stages. In some cases, patients underwent other complementary treatments such as stem cell infusion or administration of supplements. In patients harbouring tumours, GcMAF treatment was initiated at late stages of tumour progression. Therefore, since this is an open-label, non-controlled, retrospective analysis, caution must be employed when ascribing cause and effect to any treatment outcome. However, the response to GcMAF was robust and certain trends emerge evident. In all cases $(n=7)$, GcMAF subcutaneous injections were associated with improvement of clinical conditions. No adverse side effects were reported. The observation reported here confirm and extend the results previously presented by several Authors and open the way to further trials aimed at assessing the precise role and indications for GcMAF in the immunotherapy of chronic diseases.
\end{abstract}

Keywords: Cancer, Macrophages, Immunotherapy, Vitamin D, Complementary Medicine

\section{INTRODUCTION}

It is well assessed that sequential de-glycosylation of vitamin D binding protein (also termed Gc-protein) in vivo and in vitro leads to the formation of a macrophage activating factor (termed GcMAF) that exerts powerful, multiple, biological effects. Given the central role of immune system in most chronic diseases, it has been postulated that GcMAF could prove useful in a variety of conditions that are characterized by immunodeficiency or immune system dysfunction (Yamamoto et al., 2008a; 2008b; 2008c; 2009; Bradstreet et al., 2012; Inui et al., 2013). The approach of targeting the immune system in cancer and other chronic conditions has often been referred to as "immunotherapy".

Corresponding Author: Lynda Thyer, Macro Innovations Ltd, Cambridge, UK
The beneficial effects observed in the immunotherapy of human tumours (Yamamoto et al., 2008a; 2008b; 2008c; Inui et al., 2013), have been confirmed in controlled experiments performed in several laboratories, where GcMAF proved effective against tumours transplanted into animals (Toyohara et al., 2011; Nonaka et al., 2012; Hirota et al., 2013) as well as in human cancer cells cultured in vitro (Gregory et al., 2010; Pacini et al., 2012a). In addition to the effects on tumour cells, we and others demonstrated that GcMAF inhibits cancer cell-induced angiogenesis, thus depriving the tumour cell mass of the blood, oxygen and nutrient supply that it needs to grow and metastasize (Kalkunte et al., 2005; Pacini et al., 2011; 2012a; 2012b). 
Even though the majority of papers published on GcMAF so far, are in the area of clinical and experimental oncology, because of its multifaceted biological effects, GcMAF seems to be effective also in other chronic conditions that are characterized by immune dysfunction and systemic inflammation. The theoretical usefulness of GcMAF in HIV/AIDS patients was first hypothesized in 1994 (Yamamoto et al., 1995) and a study published in 2009 after prolonged follow-up, demonstrated the effectiveness of GcMAF in restoring the immune system in HIV-infected patients (Yamamoto et al., 2009).

The involvement of GcMAF in the therapy of autism is more recent and the first study on this subject was published in 2012 describing the effects of GcMAF in 40 autistic children (Bradstreet et al., 2012), whereas the effects of GcMAF in Myalgic Encephalomyelitis/Chronic Fatigue Syndrome (ME/CFS) (Carruthers et al., 2011), albeit presented at international conferences on the syndrome (Lapp, 2011) have not yet been published. The effects of GcMAF in these conditions that involve the immune system as well as the central nervous system can be ascribed to the recently elucidated GcMAF molecular signalling pathway.

Thus, we recently hypothesized that the effects of GcMAF are mediated by cross-talk with the Vitamin D Receptor (VDR) and we elucidated the molecular type of interaction occurring between these two vitamin D binding proteins that belong to the vitamin $\mathrm{D}$ axis and exert a multiplicity of effects at the cellular and molecular level (Ruggiero and Pacini, 2011; Thyer et al., 2013). Since the VDR is expressed in a variety of tissues including the central nervous system (Harms et al., 2011) it can be hypothesized that GcMAF may exert a direct effect on neurons as well as an indirect effect of rebalancing the immune system that is often dysfunctional in autism and ME/CFS.

At the molecular level, we demonstrated that GcMAF stimulates the intracellular signalling pathway belonging to the cAMP cascade and this pathway could be responsible for the apoptosis of tumour cells induced by GcMAF (Pacini et al., 2011).

The solidity of the scientific results concerning GcMAF effects in a variety of diseases and experimental systems is witnessed by the number scientific papers published by several research groups in the peerreviewed literature since the first report on GcMAF in 1994 (Yamamoto et al., 1994). Because of the solid scientific rationale underlying the compassionate use of GcMAF in patients with advanced or untreatable diseases, hundreds of physicians in all parts of the world have adopted its use in a variety of conditions where it could prove useful. In this series of clinical cases, we report some examples of the results that have been obtained administering GcMAF to patients with diverse types of cancers at advanced stages and with other diseases. In describing the following individual cases, we are well aware that these reports, because of their heterogeneity and very small numbers, can be considered anecdotes. However, it is worth noticing that a very recent study on the evaluation of clinical practice prompts for re-evaluation of individual cases such as those presented here (Nunn, 2011). Thus, it is well known that some studies present large and impressive statistics obtained from many observations while others report a small number of noteworthy events, as we do in this study. According to this novel, authoritative, epistemological approach, "all of these stories become evidence of what works in medicine" (Nunn, 2011). Therefore, we suggest that the clinical cases reported below may be considered as evidence for the effectiveness of GcMAF in different conditions and may encourage further studies on this topic.

\section{MATERIALS AND METHODS}

\subsection{GcMAF}

Physicians obtained highly purified, commercially available GcMAF from Immuno Biotech Ltd., Guernsey, Channel Islands. GcMAF was purified according to the procedure described by Yamamoto et al. (2008a). Briefly, vitamin D-binding protein was isolated from purified human serum obtained from the American Red Cross, using either 25-hydroxyvitamin D3-Sepharose high affinity chromatography, or actin-agarose affinity chromatography. The bound material was eluted and then further processed by incubation with three immobilized enzymes. The resulting GcMAF was filter sterilized. The protein content and concentration was assayed using standard Bradford protein assay methods (Bradford, 1976). At the end of the production process, GcMAF was checked for sterility "in-house" and externally by independent research laboratories. Its safety and biological activity were tested in human monocytes (Pacini et al., 2012a), human breast cancer cells (Pacini et al., 2012b) and chick embryo chorionallantoic membrane (Pacini et al., 2011). Safety and activity tests were routinely conducted on each individual batch of highly purified GcMAF. 


\subsection{Data Collection}

Data collection consisted in the recollection and analysis of the anonymized clinical records and exams sent by Physicians who had used GcMAF with their patients at the Nova Cells Institute of Mexico (NCIM, La Habra Heights, CA, USA). Administration of GcMAF to individual patients was performed by their treating Physicians according to the rules and regulations of their Country. Data collection and analysis were performed at Macro Innovations Ltd, Cambridge, UK and at the Department of Experimental and Clinical Medicine, University of Firenze, Italy. Immuno Biotech Ltd provided for data archives and bio-computational analysis support. None of the Authors of this study treated any of the patients here described.

Therefore, this study has no characteristics whatsoever of a randomized clinical trial; rather, it is a retrospective analysis of anonymised reports. The Authors left the reports as close as possible to the originals with minimal grammar and spelling corrections. Since each Practitioner used a different way of describing the condition of each individual patient, heterogeneity in the reports has to be expected.

Because of these characteristics, this study has no intent of achieving results that could be used to perform statistical analyses; the data are purposely presented as raw data so that each reader can draw her/his conclusions.

\subsection{Case Reports and Comments}

The following reports were communicated by NCIM to Immuno Biotech Ltd and refer to the years 2012-2013. In addition to GcMAF, most patients were prescribed other therapeutic approaches as indicated. GcMAF was administered by intramuscular injection following the indications outlined in the literature (Yamamoto et al., 2008a; 2008b; 2008c). The original reports are in italics.

\section{Patient ID: 001-L.R., 71 Years Male}

Cancer, prostate metastasized to the liver. Came to Nova Cells Institute of Mexico July, 2012. Both legs swollen up to the thigh. Unable to urinate. No appetite. Constipated. Extreme fatigue. Could sit down with difficulty because the prostate was enlarged the size of an orange. Lots of pain in the lower back, liver and prostate. The PSA 345. His overall colour was a pale yellow. Before he came to us he was taking antitestosterone hormone injection therapy. No chemo or radiation. Our doctors stabilized him, drawing 3 quarts of urine from his bladder. Gave him enemas and completely controlled the inflammation in both his legs. This stabilization took 7 days. He was then given our treatment which consists of 10 infusions of donor granulocytes which have been obtained by the process of apheresis from young donors, whose granulocytes are very aggressive against cancer cells. One infusion per day for 5 days then he rests 2 days and so forth until finished. The granulocytes are primed with the NCIM proprietary technology. He stayed one month. By the end of two weeks he was not jaundiced any more. He had a pink colour to his face and palms of his hands and enormous appetite. He was given GcMAF twice a week, then took the rest home. He injected himself twice a week all three vials. He is still injecting more GcMAF but only once a week. Three months after his treatment with us his PSA was 1 . He had no pain, was eating great and most astonishing his prostate had shrunken to the size of a walnut. His biopsy says no more cancer.

The results presented in this case are consistent with the original study by Yamamoto et al. (2008a) who reported that prostate cancer patients treated with purified GcMAF were "... tumour-free. No recurrence occurred for 7 years" (Yamamoto et al., 2008a). They are also consistent with the more recent report by Gregory et al. (2010) describing a direct effect of GcMAF on human prostate cancer cells with inhibition of their proliferation and metastatic potential. It should be noticed, however, that this individual patient underwent also other types of immunotherapeutic approaches and therefore it is difficult to ascertain the exact role of each therapeutic approach. This type of interpretative difficulty is common in advanced cancer patients who are often simultaneously treated with different conventional and non-conventional approaches in order to achieve the best results. For example, a very recent report describes the effects of a combined immunotherapy comprising GcMAF-containing human serum, natural killer cell therapy, intravenous high-dose vitamin $\mathrm{C}$, alpha lipoic acid and vitamin D3 administered orally (Inui et al., 2013); it is evident that also in this case it is difficult to ascertain the individual merit of each treatment.

\section{Patient ID: 002-J., 62 Years Female}

Cancer: Inflammatory Breast cancer. Was diagnosed in April of 2012. Had two rounds of chemotherapy but was having pain still. The doctors did not give her much hope. She came to NCIM in November 2012. She received the granulocyte therapy plus stem cells from umbilical cord blood that have been programmed and primed. She has been injecting GcMAF once a week, 
Her cancer titers are very low. She has no more pain. We are monitoring her. She says that she feels no more symptoms. The cancer is going in remission.

The results presented in this case are consistent with the original study by Yamamoto et al. (2008c) reporting that metastastic breast cancer patients treated with GcMAF obtained "eradication of the tumours. This therapeutic procedure resulted in no recurrence for more than 4 years." (Yamamoto et al., 2008c). They are also consistent with the results by Pacini et al. (2012b) who described direct effects of GcMAF on human breast cancer cell proliferation and metastatic potential as well as on human breast cancer cell-stimulated neoangiogenesis (Pacini et al., 2012b).

\section{Patient ID: 003-P.H., 33 Years Male}

CFS: He has been suffering from chronic fatigue syndrome for the last 14 years. Had been tested for every virus and Lymes (Borellia) but always was negative except HHV-6. The titers were 1,200 in 2011 and 2012. Otherwise very healthy. His symptoms were fatigue but not bed ridden. Only needed to take a 30 to 60 min nap once or twice a day. The immune system was quite low. The Natural Killer cells were very low. His CD3 and CD4 immune cells were very very low. He had a cold every month and sometimes the colds would last 3 months. Neutrophils, very low. Has been also diagnosed with fibromyalgia. Experiencing pain in the calves, thigh and arms as well as upper back. This, when stressed. Headaches when in the sun for a few hours. Suffered insomnia once in a while. He did not test for autoimmune disease. Started injecting GcMAF in October of 2012. He started not being tired after 16 weeks of therapy. He only gets somewhat tied every 2-3 weeks and just takes a 15 min nap and feels great. Only when stressed. After he finished the fourth GcMAF vial, his fibromyalgia pains are all gone. The HHV-6 titers are nonexistent. The NK cell as well as the CD 3 and 4's are above normal. He is going to continue with the GcMAF injections until he finishes the 6 vials. He is ecstatic and so are we.

The results presented in this case are consistent with those presented at the http://www.research1st.com/wpcontent/uploads/2011/10/IACFS.jpgbiennial meeting of the IACFS/ME held in Ottawa, Ontario, in 2011 (http://www.research1st.com/2011/10/13/iacfsme-

summary/). They are also consistent with the very recent publication by Meirleir et al. (2013) demonstrating an association between human endogenous retroviruses and ME/CFS (Meirleir et al., 2013). Therefore, the results observed in this patient could be ascribed both to a reinforcement of the immune system with consequent improved control on viral infections and/or to a possible direct effect of GcMAF on neurons through cross-talk between GcMAF signalling and the VDR.

\section{Patient ID: 004-C.Y., 35 Years Female}

Multiple Sclerosis, with Lymes (Borellia). In wheelchair for 7 years. Can not walk. Not to alert. Could not carry on a conversation well. Distracted. Twitches, stiffness. We have given her two treatments of cord blood stem cells. One every 7 months. She showed hardly any benefit with the first treatment but in October we added GcMAF to the treatment. She is doing amazing. She can be understood on the phone. The mother is taking her to her business and is conversing with the staff. I personally spoke to her 4 days ago. She carried on a conversation which she could not before. She started raising one leg then the other while lying in bed. Then steps with help. She is now standing by herself for almost an hour just helping herself holding on to a chair. One other symptom that she had was excessive shaking when she was stressed if people looked at her. This has almost disappeared. She is eating very well and is putting on make up. The Lymes is not showing in her test anymore.

To our current knowledge, the results presented in this case have not yet been reported in other publications. It can be hypothesized that they can be ascribed to the stimulation of the immune system by GcMAF.

\section{Patient ID: 005-A.K., 67 Years Male}

Prostate Cancer: Metastasized to the liver, bone and brain. Jaundiced. The Alkaline Phosphatase was 398 from the bone cancer. The PSA 1,600. The prostate as large as a grapefruit. Came to us in October 2012. He was treated with then rounds of granulocytes by apheresis and stem cells from the blood of three umbilical cords, Myschemals and CD34's as well as other more primitive stem cells derived from the Wharton's jelly of the three cords. He stayed in Mexico one month. Was detoxed, stabilized, given organic juices, choldronate to avoid more bone metastazis. He was given GcMAF. 50 injections twice a week until he left. He has been on the cancer protocol ever since and taking the GcMAF in synergy with the our cancer supplement protocol. We have sent him more MAF to continue the treatment. I have finally spoken to the family last week after trying to reach them in Russia for a couple of weeks. His liver is clearing the cancer. The brain tumour is shrinking. The prostate is shrinking. He is alive and plans to continue with his GcMAF 
injections. He did have a clot in his leg but were able to take it out. His alkaline Phosphatase is now 80 because there is less bone destruction from the bone cancer. His PSA is 45 since last week.

The results presented in this case are consistent with those observed in case \#1. Also in this case the patient underwent also other types of non-conventional approaches and therefore it is difficult to ascertain the exact role of each approach.

\section{Patient ID: 006-H., 38 Years Male}

ALS or ALS-like symptoms (with Syphilis for 12 years without knowing). He started having symptoms in December 2010. He started not being able to button his shirt buttons. Then unable to move his arms and not able to feed himself or go to the bathroom by himself. He then was not able to eat anything without drinking a fourth glass of water with each bite of food. He needed help climbing stairs so family members had to drag him upstairs to his room. He started choking on his food and was using a respirator off and on. We gave him autologous bone marrow stem cells that were programmed and stem cells from cord blood. He was treated with different antibiotics against the syphilis and given GcMAF. After the second stem cell treatment 30 days later, he was able to hold an ice cream cone and feed himself. After the first stem cell treatment and continuing with his GcMAF treatment, he was walking with a cane and some times with help. 6 months after his treatments he started climbing up and down stairs just holding on to the rail. Then started doing work-outs on his treadmill. He is stable up until last week. He is not progressing. There is no more syphilis.

The improvement of this patient can be ascribed to a combination of effects. The stimulation of the immune system by GcMAF could be responsible for the improvement of the infectious aspects of this complex situation. Also a direct effect of GcMAF on neurons cannot be ruled out.

\section{Patient ID: 007 - J.S., 11 Years Male}

Autistic. Would not play with other children. Would never look at people in the eyes. Withdrawn. Talked to himself mostly. Said incoherent things. As well all many other symptoms of autism.

Started GcMAF treatment December 2012. After he had finished 12 weeks he started communicating with his mother. He is interacting with other children. $\mathrm{He}$ is not talking to himself very much. He is reciprocating and accepting hugs.
The results presented in this case are fully consistent with those published by Bradstreet et al. (2012) who reported that "GcMAF therapy indicated substantial improvements in language, socialization and cognition" in autistic children.

\section{RESULTS AND DISCUSSION}

It is well assessed that GcMAF is effective against a variety of experimental and spontaneous tumours as well as against various aspects of neoplastic transformation in vitro (Yamamoto et al., 2008a; 2008b; 2008c; Nonaka et al., 2012; Gregory et al., 2010; Pacini et al., 2012b). The clinical cases reported here are heterogeneous and describe patients with different types of tumours at different stages as well as patients with other pathologies. In many cases, patients underwent other treatments. In all cancer cases, GcMAF treatment was initiated at late stages of tumour progression since it is understandable that conventional therapies were preferred at earlier stages. In addition, since this is an open-label, non-controlled, retrospective analysis, caution must be employed when ascribing cause and effect to any treatment outcome. However, the response to GcMAF was robust and, even though any statistical analysis is inappropriate in such a heterogeneous recollection of clinical stories, the clinical improvement appears to be evident.

A significant point that emerges from the study of the cases described above is the absence of any reported side effects associated with GcMAF injections in patients. This point, previously assessed also in autistic children (Bradstreet et al., 2012), assumes great importance when considering the administration of GcMAF in the context of the so called compassionate treatment of patients with advanced or incurable diseases. In fact, in many countries the absence of side effects is a pre-requisite for the compassionate administration of substances that have not yet been approved by local health authorities. It is obvious that these preliminary reports need a much longer follow-up in order to assess the best indications for GcMAF treatment.

For example, referring to the role of GcMAF in oncology, GcMAF has been used, with encouraging results, in all types of cancers at all stages by physicians who use it as compassionate treatment. However, it can be hypothesized that certain histological types as well as certain definite stages of cancer might have a differential response to GcMAF. Also the patient's genotype as far 
as VDR gene polymorphisms are concerned, might influence the individual response to GcMAF. In fact, it was recently demonstrated that the degree of the response of normal human monocytes to GcMAF is associated with individual VDR genotypes (Pacini et al., 2012a) and it can be hypothesized that also the response in terms of anti-neoplastic effects may be associated with such polymorphisms. Finally, it should be remembered that the prognosis for all types of cancers is dependent upon the nutritional and inflammatory status of the patient that can be monitored by the Prognostic Inflammatory and Nutritional Index (PINI) (Fabris et al., 2012). The PINI score therefore could become part of the laboratory assessment during GcMAF treatment and together with Nagalase testing and VDR gene polymorphism determination, it could help monitor the response of each individual patient allowing adjustment of the dose and the frequency of administration in order to obtain the optimum results.

The preliminary results reported here are consistent with the recent publication of Inui et al. (2013) who described three clinical cases successfully treated with a combination of therapies including subcutaneous or intramuscular injections of GcMAF-containing human serum (Hirota et al., 2013). However, at variance with their study, the results presented here were obtained with highly purified GcMAF instead of injecting human serum which was not purified using a vitamin D affinity column (Hirota et al., 2013). Therefore, we can conclude that the results that we observed are to be attributed to the highly purified GcMAF used in this study and not to other serum proteins that might have acted as confounding factors.

\subsection{Potential Conflicts of Interest}

$\mathrm{DN}$ is the CEO of Immuno Biotech, Ltd (the company isolating and purifying the GcMAF protein). However, DN had no knowledge of the therapies being used nor of the names of any patients whose data were being analyzed. Neither he, nor any employee of Immuno Biotech, Ltd had any knowledge of the clinical records or the patient names used in this study.

\section{CONCLUSION}

The observations reported here confirm and extend the results presented by several Authors on the role of GcMAF in different conditions and open the way to further studies aimed at assessing the precise role and indications of GcMAF in the immunotherapy of cancer and other chronic diseases.

\section{REFERENCES}

Bradford, M.M., 1976. A rapid and sensitive method for the quantitation of microgram quantities of protein utilizing the principle of protein-dye binding. Analytical Biochem., 72: 248-254. PMID: 942051

Bradstreet, J.J., E. Vogelaar and L. Thyer, 2012. Initial observations of elevated alpha-Nacetylgalactosaminidase activity associated with autism and observed reductions from GC proteinmacrophage activating factor injections. Autism Insights, 4: 31-38. DOI: 10.4137/AUI.S10485

Carruthers, B.M., M.I.V. de Sande, K.L. De Meirleir, N.G. Klimas and G. Broderick et al., 2011. Myalgic encephalomyelitis: International consensus criteria. J. Int. Med., 270: 327-338. DOI: 10.1111/j.13652796.2011.02428.x

Fabris, A., P. Biagioni, T. Punzi, G. Morucci and M. Gulisano et al., 2012. Role of angiotensinconverting enzyme and vitamin D receptor gene polymorphisms in cancer anorexia-cachexia syndrome. Am. J. Immunol., 8: 65-70. DOI: 10.3844/ajisp.2012.65.70

Gregory, K.J., B. Zhao, D.R. Bielenberg, S. Dridi and J. $\mathrm{Wu}$ et al., 2010. Vitamin D binding proteinmacrophage activating factor directly inhibits proliferation, migration and UPAR expression of prostate cancer cells. PLoS One, 5: e13428. DOI: 10.1371/journal.pone. 0013428

Harms, L.R., T.H. Burne, D.W. Eyles and J.J. McGrath, 2011. Vitamin D and the brain. Best Practice Res. Clin. Endocrinol. Metab., 25: 657-669. DOI: 10.1016/j.beem.2011.05.009.

Hirota, K., Y. Nakagawa, R. Takeuchi, Y. Uto and H. Hori et al., 2013. Antitumor effect of degalactosylated gc-globulin on orthotopic grafted lung cancer in mice. Anticancer Res., 33: 29112915. PMID: 23780979

Inui, T., D. Kuchiike, K. Kubo, M. Mette and Y. Uto et al., 2013. Clinical Experience of integrative cancer immunotherapy with GcMAF. Anticancer Res., 33: 2917-2919. PMID: 23780980

Kalkunte, S., L. Brard, C.O. Granai and N. Swamy, 2005. Inhibition of angiogenesis by vitamin Dbinding protein: Characterization of anti-endothelial activity of DBP-maf. Angiogenesis, 8: 349-360. DOI: $10.1007 / \mathrm{s} 10456-005-9024-7$

Lapp, C., 2011. IACFS/ME International Conference Summary. Hunter-Hopkins Center, P.A., Charlotte, North Carolina. 
Meirleir, D., K.L.S.F. Khaiboullina, M. Fremont, J. Hulstaert and A.A. Rizvanov et al., 2013. Plasmacytoid dendritic cells in the duodenum of individuals diagnosed with myalgic encephalomyelitis are uniquely immunoreactive to antibodies to human endogenous retroviral proteins. In Vivo, 27: 177-187. PMID: 23422476

Nonaka, K., S. Onizuka, H. Ishibashi, Y. Uto and H. Hori et al., 2012. Vitamin D binding proteinmacrophage activating factor inhibits HCC in SCID mice. J. Surgical Res., 172: 116-122. DOI: 10.1016/j.jss.2010.07.057

Nunn, R., 2011. Mere anecdote: Evidence and stories in medicine. J. Eval. Clin. Practice, 17: 920-926. DOI: 10.1111/j.1365-2753.2011.01727.x

Pacini, S., G. Morucci, T. Punzi, M. Gulisano and M. Ruggiero et al., 2012a. Effect of paricalcitol and GcMAF on angiogenesis and human peripheral blood mononuclear cell proliferation and signaling. J. Nephrol., 25: 577-581. DOI: 10.5301/jn.5000035

Pacini, S., G. Morucci, T. Punzi, M. Gulisano and M. Ruggiero, 2011. Gc protein-derived MacrophageActivating Factor (GcMAF) stimulates cAMP formation in human mononuclear cells and inhibits angiogenesis in chick embryo chorionallantoic membrane assay. Cancer Immunol. Immunotherapy, 60: 479-485. DOI: 10.1007/s00262-010-0953-7

Pacini, S., T. Punzi, G. Morucci, M. Gulisano and M. Ruggiero, 2012b. Effects of vitamin D-binding protein-derived macrophage-activating factor on human breast cancer cells. Anticancer Res., 32: 45 52. PMID: 22213287

Ruggiero, M. and S. Pacini, 2011. The vitamin D axis in chronic kidney disease-state of the art and future perspectives. Eur. Nephrol., 5: 15-19.

Thyer, L., E. Ward, R. Smith, M.G. Fiore and S. Magherini et al., 2013. A novel role for a major component of the vitamin D axis: Vitamin D binding protein-derived macrophage activating factor induces human breast cancer cell apoptosis through stimulation of macrophages. Nutrients, 5: 2577-2589. DOI: $10.3390 /$ nu5072577
Toyohara, Y., S. Hashitani, H. Kishimoto, K. Noguchi and N. Yamamoto et al., 2011. Inhibitory effect of vitamin D-binding protein-derived macrophage activating factor on DMBA-induced hamster cheek pouch carcinogenesis and its derived carcinoma cell line. Oncol. Lett., 2: 685-691. DOI: 10.3892/ol.2011.306

Yamamoto, N., D.D. Lindsay, V.R. Naraparaju, R.A. Ireland and S.N. Popoff, 1994. A defect in the inflammation-primed macrophage-activation cascade in osteopetrotic rats. J. Immunol., 152: 5100-5107. PMID: 8176226

Yamamoto, N., H. Suyama and N. Yamamoto, 2008a. Immunotherapy for prostate cancer with gc proteinderived macrophage-activating factor, GcMAF. Trans. Oncol., 1: 65-72. DOI 10.1593/tlo.08106

Yamamoto, N., H. Suyama, H. Nakazato, N. Yamamoto and Y. Koga, 2008b. Immunotherapy of metastatic colorectal cancer with vitamin D-binding proteinderived macrophage-activating factor, GcMAF. Cancer Immunol. Immunotherapy, 57: 1007-1016. DOI: $10.1007 / \mathrm{s} 00262-007-0431-\mathrm{z}$

Yamamoto, N., H. Suyama, N. Yamamoto and N. Ushijima, 2008c. Immunotherapy of metastatic breast cancer patients with vitamin D-binding protein-derived Macrophage Activating Factor (GcMAF). Int. J. Cancer, 122: 461-467. DOI: $10.1002 /$ ijc. 23107

Yamamoto, N., N. Ushijima and Y. Koga, 2009. Immunotherapy of HIV-infected patients with Gc protein-derived Macrophage Activating Factor (GcMAF). J. Med. Virol., 81: 16-26. DOI: 10.1002/jmv.21376

Yamamoto, N., V.R. Naraparaju and S.M. Srinivasula, 1995. Structural modification of serum vitamin D3binding protein and immunosuppression in AIDS patients. AIDS Res. Hum. Retroviruses, 11: 13731378. DOI: 10.1089/aid.1995.11.1373 